\title{
Balloon Tamponade With Sengstaken-Blakemore Tube for Penetrating "Core" Liver Injury
}

\author{
Alexander Becker ${ }^{\mathrm{a}, \mathrm{c}}$, Hagar Mizrahia ${ }^{\mathrm{a}}$, Alexander Chulsky ${ }^{\mathrm{b}}$
}

\begin{abstract}
Gunshot injuries to the liver present challenging management problems. Abdominal packing is a useful and widely accepted technique to achieve hemostasis in complex hepatic injury, but packing alone may not provide adequate hemostasis, especially in active arterial bleeding from central liver gunshot wound. We present case report describes the use of a Sengstaken-Blakemore tube with concomitant angiography to control exsanguinating bleeding from a central hepatic gunshot injury. Balloon tamponade followed by angioembolisation is a highly successful option for central hepatic gunshot injury and should be in armamentarium of trauma surgeon.
\end{abstract}

Keywords: Liver injury; Balloon tamponade; Angioembolisation

\section{Introduction}

Gunshot injuries to the liver present challenging management problems. Abdominal packing is a useful and widely accepted technique to achieve hemostasis in complex hepatic injury, but packing alone may not provide adequate homeostasis, especially in active arterial bleeding from central liver gunshot wound. Angiography can also be used successfully to achieve hemostasis in active arterial hemorrhage, but often is not readily available in exsanguinating patients, where immediate bleeding control is life saving. The use of balloon catheter tamponade for arresting ongoing hemorrhage from the central liver is well described [1-3]. This case re-

Manuscript accepted for August 8, 2012

\footnotetext{
${ }^{a}$ Department of Surgery A, Haemek Medical Center, Afula, B. Rappaport School of Medicine, Technion, Haifa, Israel

${ }^{b}$ Department of Invasive Radiology, Haemek Medical Center, Afula, B. Rappaport School of Medicine, Technion, Haifa, Israel

${ }^{\mathrm{c} C o r r e s p o n d i n g ~ a u t h o r: ~ A l e x a n d e r ~ B e c k e r, ~ D e p a r t m e n t ~ o f ~ S u r g e r y ~ A, ~}$ Haemek Medical Center, Afula, Israel.

Email: alexb20042000@yahoo.com
}

doi: http://dx.doi.org/10.4021/jcs94w port describes the use of a Sengstaken-Blakemore tube with concomitant angiography to control exsanguinating bleeding from a central hepatic injury.

\section{Case Report}

A 22-year-old male sustained multiple gunshot wounds to his abdomen. On arrival, he was hemodynamically unstable with a systolic blood pressure of 85 and a heart rate of 132 . Chest radiography showed no abnormalities. The patient's abdomen was tender to palpation in the upper quadrants.

Immediate laparotomy revealed two liters of blood in the abdominal cavity, active bleeding from a thru-and-thru liver injury. One wound was located in the left lobe anteriorly, with a tract of approximately $20 \mathrm{~cm}$ in length, towards the posterior surface of the right lobe; a second wound was located in the posterior lateral aspect of the right lobe. A laceration of the right diaphragm was found. No other intraabdominal injuries were noted.

Despite liver mobilization with subsequent packing and an attempted Pringle maneuver, a significant amount of bleeding was observed through the wounds. A SengstakenBlakemore tube was placed through the wound to the depth of the liver. The esophageal balloon was inflated with air to

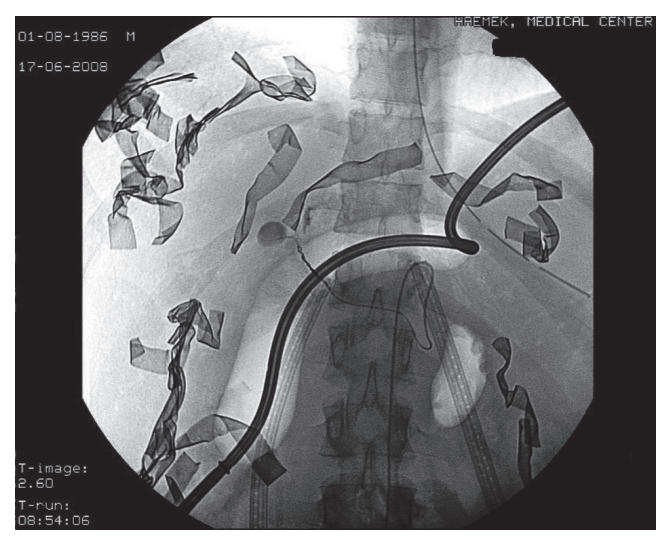

Figure 1. Angiography revealed active arterial bleeding from a branch of the right hepatic artery. Site-specific angio-embolization with metal coil and gel foam was performed. 


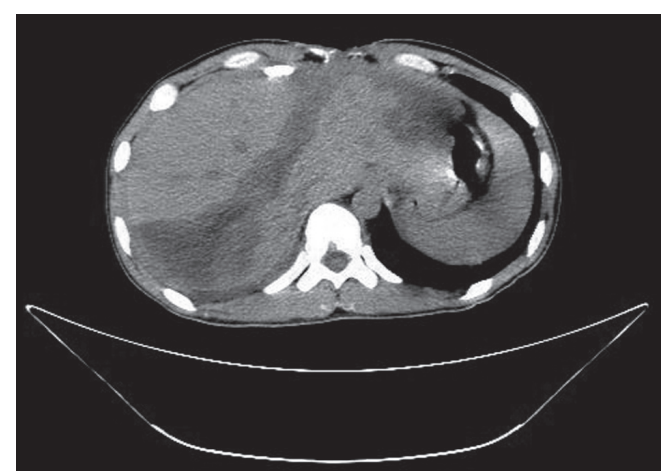

Figure 2. A CT scan obtained 1 week postoperatively demonstrated the long bullet tract of "core "liver injury.

tamponade the bullet tract (Fig. 1). This maneuver significantly decreased the bleeding but did not completely stop it. The gauze packs were left in place, the diaphragm laceration was sutured and temporary abdominal closure with vacuum pack system was performed. A right chest tube was inserted with an initial output of $250 \mathrm{~mL}$ of blood. Throughout the surgery the patient was transfused with $6 \mathrm{~L}$ of Ringer Lactate. Massive blood transfusion protocol was activated and the patient was given 6 PRBC, 6 FFP, platelets and cryoprecipitate.

Following surgery the patient was immediately taken to angiography, which revealed active arterial bleeding from a branch of the right hepatic artery (Fig.1). Site-specific angioembolization with metal coil and gel foam caused bleeding to cease. A second -look laparotomy was performed at 36 hours on a hemodynamically stable patient. The balloon was deflated and removed without evidence of further bleeding. The patient's abdomen was then closed with a vicryl mesh and two closed suction perihepatic drains were left in place. After surgery, bile leakage subsided and completely stopped on day 6 . The operative wound was closed with a skin graft and the patient was discharged home on day 22 of his hospitalization. A CT scan obtained 1 week postoperatively for fever evaluation demonstrated the long bullet tract (Fig. 2).

\section{Discussion}

Penetrating central injuries to the liver can be very challenging in a patient in extremis who requires immediate bleeding control as part of damage control laparotomy. Different techniques, such as hepatorrhaphy, resection and debridement, tractotomy with suture ligation of the bleeders, hepatic artery ligation, are often applied but have limited success and may even exacerbate bleeding.

The use of a balloon catheter for bleeding control from the central liver is a well established technique [4]. Although different devices, such as red rubber catheter and Penrose drain or a Foley catheter, have been proposed for balloon tamponade, a Sengstaken-Blakemore tube has the advantage of being available for immediate use. The use of balloon catheter tamponade to achieve hemostasis in penetrating "core" liver injuries manages to control bleeding in $83 \%$ of patients [5]. In this case, it did not stop bleeding completely and the patient underwent therapeutic angiography with embolisation. This is a very important adjunct and should always be strongly considered when complete bleeding control is not achieved, or in cases of re-bleeding after removal of an indwelling catheter.

When the usual surgical techniques described above are either ineffective or inappropriate, balloon tamponade followed by angioembolisation should be considered a highly successful option for core hepatic gunshot wounds, and should be an integral part of the trauma surgeon's tools.

\section{Conflict of Interest Statement}

We certify that there are no any relevant financial interests or financial conflicts, at the time the research was conducted and up to the time of publication, as well as other financial interests, such as relevant filed or pending patents, that represent potential future financial gain.

\section{References}

1. Poggetti RS, Moore EE, Moore FA, Mitchell MB, Read RA. Balloon tamponade for bilobar transfixing hepatic gunshot wounds. J Trauma. 1992;33(5):694-697.

2. Thomas SV, Dulchavsky SA, Diebel LN. Balloon tamponade for liver injuries: case report. J Trauma. 1993;34(3):448-449.

3. Demetriades D. Balloon tamponade for bleeding control in penetrating liver injuries. J Trauma. 1998;44(3):538539.

4. Fabian TC, Bee TK. Liver and biliary tract. In: Feliciano DV, Mattox KL, Moore EE, eds. Trauma. 6th ed. New York: McGraw-Hill Medical; 2008:637-660.

5. Ball CG, Wyrzykowski AD, Nicholas JM, Rozycki GS, Feliciano DV. A decade's experience with balloon catheter tamponade for the emergency control of hemorrhage. J Trauma. 2011;70(2):330-333. 